\title{
COINCIDENCE FUNCTIONS AND THEIR INTEGRALS
}

\section{BY \\ HERBERT FEDERER}

1. Introduction. Two functions $f$ and $g$ are said to coincide at $(x, y)$ if and only if $f(x)=g(y)$. If $f$ parametrizes a $k$-dimensional surface in Euclidean $n$-space, and $g$ an $(n-k)$-dimensional surface, then the number of coincidences of $f$ and $g$ is the number of intersections of these surfaces.

Now keep the first surface fixed and move the second rigidly; or, otherwise said, superimpose upon $g$ an arbitrary isometric transformation, $S$, of $n$-space. Count the number of coincidences of $f$ with the superposition $(S: g)$, that is, the number of intersections of the fixed and the movable surface. Integrate this count over the group of all isometric transformations of $n$-space with respect to its Haar measure, properly and explicitly normalized. If $f$ and $g$ are sufficiently regular, say Lipschitzian, the value of the integral is the product of the areas of the two surfaces times a number, $\beta(n, k)$, which depends only on the dimensions $n$ and $k$.

Poincare proved this for two curves in the plane, the special case $n=2$, $k=n-k=1$ (see $[\mathrm{P}]$ )( ${ }^{1}$ ). F.or a curve and a line segment it had previously been obtained by Crofton. The "integral geometry" of Blaschke and his students is related to the problem (see [B]). In fact Santaló obtained our result for a curve and an ordinary surface in 3-space, the special case $n=3, k=1$, $n-k=2$ (see [S]).

The general result described above is established in this paper, and by methods which we believe new and of interest even for known special cases.

These methods also lead to an extension of Cauchy's formula for the area of a convex surface: The area of a sufficiently regular $k$-dimensional surface in $n$-space equals $\beta(n, k)^{-1}$ times the average area of its projection into a $k$-dimensional subspace of $n$-space. This, in turn, suggests the definition of a lower semi-continuous area for all continuous $k$-dimensional surfaces in terms of the stable values of their projections into $k$-dimensional subspaces. This area has obvious technical advantages over those of Peano and Geöcze (see [R1]). Its relation to Lebesgue area is only partially settled.

In addition, this paper raised certain problems (see 4.7), whose consideration led us to obtain the following result (see 4.8): The Gauss-Green theorem holds for every bounded open subset of Euclidean $n$-space, whose boundary has finite $(n-1)$-dimensional Hausdorff measure. This proposition supplements the results of our recent paper, $[\mathrm{F} 3]$, on the subject. The questions raised in 4.7 will be discussed further in a forthcoming paper.

Presented to the Society, April 28, 1945; received by the editors April 11, 1945.

(1) Symbols in brackets refer to the Bibliography at the end of the paper. 
2. Notation. The terminology of this paper is consistent with that of [F1] and [F2]. We therefore refer the reader to the introductory sections of these papers for a fuller explanation of some notational details.

Some (though not all) of these notations are, however, repeated here for the reader's convenience:

$f^{*}(S)$ is the image of the set $S$ under the function $f$.

$N(f, S, y)$ is the number (possibly $\infty$ ) of points $x$ in $S$ for which $f(x)=y$.

The Lebesgue integral of the function $f$ over the set $S$ with respect to the measure $\phi$ is denoted by

$$
\int_{S} f(x) d \phi x
$$

We write $\operatorname{det} A$ for the determinant of the square matrix $A$.

We make no distinction between a linear function, $L$, and its matrix. If $L$ has $p$ columns and $q$ rows, then the $j$ th column (here $j=1,2, \cdots, p$ ) of $L$ is a point of $q$-space, $E_{q}$, and is denoted by $L^{i}$.

If $p \leqq q$, then

$$
\Delta(L)=\left\{\sum_{x \in S}\left(\operatorname{det} M_{x}\right)^{2}\right\}^{1 / 2},
$$

where $x \in S$ if and only if $x$ is a set of $p$ integers between 1 and $q$; and $M_{x}$ is the minor of $L$ which is made up from the $p$ rows whose indices are elements of $x$.

If the function $f$ on $E_{p}$ to $E_{q}$ has at the point $x$ in $E_{p}$ the approximate differential $L$, then we denote

$$
J f(x)=\Delta(L) .
$$

For use in the present paper, we next introduce the following notations:

2.1. Definition. If $f$ and $g$ are functions, and $U$ and $V$ are sets, then

$$
\Omega(f, U ; g, V)
$$

is the number (possibly $\infty$ ) of ordered pairs $(u, v)$ for which $u \in U, v \in V$ and $f(u)=g(v)$.

2.2. Definition. $E_{n}$ is Euclidean $n$-space;

$$
\begin{array}{rlrl}
x & =\left(x_{1}, x_{2}, \cdots, x_{n}\right) & \text { for } x & \in E_{n} ; \\
x \cdot y & =\sum_{i=1}^{n} x_{i} y_{i} & \text { for } x \in E_{n}, y \in E_{n} .
\end{array}
$$

2.3. Definition. If $x \in E_{k}$ and $y \in E_{m}$, then

$$
(x \circ y)=\left(x_{1}, x_{2}, \cdots, x_{k}, y_{1}, y_{2}, \cdots, y_{m}\right) \in E_{k+m} .
$$

If $f$ is a function on $E_{k}$ to $E_{n}$, and $g$ is a function on $E_{m}$ to $E_{n}$, then

$$
(f \ominus g)
$$


is the function on $E_{k+m}$ to $E_{n}$ such that

$$
(f \ominus g)(x \circ y)=f(x)-g(y) \text { whenever } x \in E_{k}, y \in E_{m} .
$$

2.4. Definition. If $m \leqq n$ are positive integers, then $\mathcal{L}_{n}{ }^{m}$ is the $m$-dimensional measure over $E_{n}$ defined, as $\Phi$, in $[\mathrm{F} 2,2.1]$.

Hence the $n$-dimensional Lebesgue measure of $X \subset E_{n}$ is $\mathcal{L}_{n}{ }^{n}(X)$, and we shall not denote it by $|X|$ in this paper.

2.5. Definition. For $n=1,2,3, \cdots$ let

$$
\alpha(n)=\mathcal{L}_{n}^{n}\left\{E_{n} \underset{x}{E}[|x|<1]\right\} .
$$

It is well known that

$$
\begin{gathered}
\alpha(n)=\left(2^{n} / n !\right) \Gamma(1 / 2)^{n-1} \Gamma((n+1) / 2), \\
\mathcal{L}_{n}^{n-1}\left\{E_{n} \underset{x}{E}[|x|=1]\right\}=n \alpha(n) .
\end{gathered}
$$

2.6. Definition. Suppose $f$ is a function on a subset of $E_{k}$ to $E_{n}$. Then $f$ is Lipschitzian on $X$ if and only if there is such a number $A$ that

$$
|f(x)-f(y)| \leqq A|x-y| \quad \text { for } x \in X, y \in X .
$$

Further, $f$ is countably Lipschitzian on $X$, if and only if $X$ is the countable sum of sets on each of which $f$ is Lipschitzian.

2.7. Definition. $M_{n}{ }^{k}$ is the set of all linear transformations on $E_{k}$ to $E_{n}$, or, otherwise said, of all real matrices with $n$ rows and $k$ columns (see [F2, 3.1]).

If $A \in M_{n}{ }^{m}$, and $k \leqq m, r \leqq n$ are positive integers, then

$$
\left(A \mid \begin{array}{l}
k \\
r
\end{array}\right)
$$

is the matrix in $M_{r}^{k}$ such that

$$
\left(\left.A\right|_{r} ^{k}\right)_{i}^{j}=A_{i}^{j} \quad \text { for } i=1,2, \cdots, r ; j=1,2, \cdots, k .
$$

Thus $\left(A \mid r^{k}\right)$ is the upper left-hand minor of $A$ with $r$ rows and $k$ columns.

We further define

$$
P_{A}^{k}=\left(\left.A\right|_{k} ^{n}\right) \quad \text { for } A \in M_{n}^{n} ; k=1,2, \cdots, n .
$$

The projecting functions of $[\mathrm{F} 2,2.1]$ are exactly the functions $P_{A^{k}}$ with $A$ orthogonal.

2.8. REMARK. We shall also use the definitions of $[F 1, \S 2]$ and of $[F 2, \S 3]$.

3. The invariant integral for the group of distance preserving transformations of $n$-space. Let $K_{n}$ be the set of all distance preserving transformations of $n$-space. Thus $f \in K_{n}$ if and only if $f$ is such a function on $E_{n}$ to $E_{n}$ that 


$$
|f(x)-f(y)|=|x-y| \quad \text { whenever } x \in E_{n} \text { and } y \in E_{n} .
$$

Next let

$$
G_{n}=K_{n} E[f(\theta)=\theta],
$$

the set of all orthogonal transformations of $n$-space. Here $\theta$ is the zero of $n$-space.

The translation $T_{z} \in K_{n}$ is associated with $z \in E_{n}$ by the relation

$$
T_{z}(x)=z+x \quad \text { for } x \in E_{n} .
$$

Recall that $K_{n}$ and $G_{n}$ are groups with respect to the operation, :, of surperposition, and that each element $S$ of $K_{n}$ has a unique decomposition

$$
S=\left(T_{z}: R\right) \quad \text { with } z \in E_{n} \text { and } R \in G_{n} \text {. }
$$

Suppose $K_{n}$ is so metrized that the group operation, :, is continuous in the corresponding topology, and that $K_{n}$ is a boundedly compact, and $G_{n}$ a compact, topological group.

In view of its compactness, $G_{n}$ carries exactly one Haar measure, $\phi_{n}$, for which $\phi_{n}\left(G_{n}\right)=1$.

There are many Haar measures-or invariant integrals-over $K_{n}$, any two of which differ by a constant factor. In selecting a particular one for the use of this paper, we are guided by these facts:

The group of translations is isomorphic with the vector space $E_{n}$; it is an invariant subgroup of $K_{n}$; its factor group is isomorphic with $G_{n}$.

This leads (see $[\mathrm{W}, 9]$ ) to the definition

$$
M_{n}(f)=\int_{G_{n}} \int_{E_{n}} f\left(T_{z}: R\right) d \mathcal{L}_{n}^{n} z d \phi_{n} R
$$

for each continuous function $f$ on $K_{n}$, which vanishes outside some compact set. It can readily be verified that the functional $M_{n}$ is an invariant integral for the group $K_{n}$.

3.1. Definition. If $k \leqq n$ are positive integers, then

$$
\beta(n, k)=\int_{G_{n}} \Delta\left(\left.R\right|_{k} ^{k}\right) d \phi_{n} R .
$$

Clearly $\beta(k, k)=1$. In $\S 5$ we shall evaluate $\beta(n, k)$ for $k<n$.

3.2. REMARK. For convenience we state here explicitly the two main properties of $\phi_{n}$ :

If $f$ is a $\phi_{n}$ measurable function on $G_{n}$, then $S \in G_{n}$ implies

and

$$
\int_{G_{n}} f(S: R) d \phi_{n} R=\int_{G_{n}} f(R: S) d \phi_{n} R=\int_{G_{n}} f(R) d \phi_{n} R
$$




$$
\int_{G_{n}} f\left(R^{\prime}\right) d \phi_{n} R=\int_{G_{n}} f(R) d \phi_{n} R,
$$

where $R^{\prime}$ is the inverse of the orthogonal matrix $R$.

These propositions follow from the fact that $G_{n}$ is compact, hence unimodular. (See $[\mathrm{W}, 8]$.)

\section{The main theorems.}

4.1. Lemma. If $A \in M_{n}{ }^{k}$ and $B \in M_{n}^{n-k}$, then

$$
\int_{G_{n}} \Delta[(R: A) \ominus B] d \phi_{n} R=\beta(n, k) \cdot \Delta A \cdot \Delta B .
$$

Proof. Select members $U$ and $V$ of $G_{n}$ such that

$$
\begin{array}{rlrl}
{\left[U\left(A^{j}\right)\right]_{i}=U_{i}\left(A^{j}\right)=0} & \text { for } & i=k+1, k+2, \cdots, n ; j=1,2, \cdots, n ; \\
{\left[V\left(B^{j}\right)\right]_{i}=V_{i}\left(B^{j}\right)=0} & \text { for } i=1,2, \cdots, k ; j=1,2, \cdots, n .
\end{array}
$$

The remainder of the proof is divided into two parts.

Part 1. If $R \in G_{n}$, then

$$
\Delta[(R: A) \ominus B]=\Delta[(V: R: A) \theta(V: B)]
$$

and

$$
\Delta[(R: U: A) \theta(V: B)]=\Delta\left(\left.R\right|_{k} ^{k}\right) \cdot \Delta A \cdot \Delta B .
$$

Proof. $\Delta[(R: A) \ominus B]=\Delta V \cdot \Delta[(R: A) \ominus B]=\Delta\{V:[(R: A) \ominus B]\}$ $=\Delta[(V: R: A) \Theta(V: B)]$.

This proves the first statement.

Next let

$$
X=(U: A), \quad Y=(V: B)
$$

and use the definition of $U$ and $V$ to check that

$$
\begin{aligned}
& X_{i}^{j}=0 \quad \text { for } \quad i=k+1, k+2, \cdots, n ; j=1,2, \cdots, k \text {; } \\
& Y_{i}^{j}=0 \quad \text { for } \quad i=1,2, \cdots, k ; j=1,2, \cdots, n-k \text {. }
\end{aligned}
$$

Hence, for $i=1,2, \cdots, k$ and $j=1,2, \cdots, k$, we have

$$
\begin{aligned}
{\left[\left.(R: X)\right|_{k} ^{k}\right]_{i}^{j} } & =(R: X)_{i}^{j}=\sum_{m=1}^{n} R_{i}^{m} X_{m}^{j}=\sum_{m=1}^{k} R_{i}^{m} X_{m}^{j} \\
& =\sum_{m=1}^{k}\left(\left.R\right|_{k} ^{k}\right)_{i}^{m} \cdot\left(\left.X\right|_{k} ^{k}\right)_{m}^{j}=\left[\left(R \mid \begin{array}{l}
k \\
k
\end{array}\right):\left(X \mid \begin{array}{l}
k \\
k
\end{array}\right)\right]_{i}^{j}
\end{aligned}
$$

Consequently

$$
\left[(R: X) \mid \begin{array}{l}
k \\
k
\end{array}\right]=\left[\left(R \mid \begin{array}{l}
k \\
k
\end{array}\right):\left(X \mid \begin{array}{l}
k \\
k
\end{array}\right)\right]
$$


From all this and $[F 2,4.2]$ we conclude

$$
\begin{aligned}
\Delta[(R: X) \ominus Y] & =\Delta\left[(R: X) \mid \begin{array}{l}
k \\
k
\end{array}\right] \cdot \Delta Y \\
& =\Delta\left(\left.R\right|_{k} ^{k}\right) \cdot \Delta\left(X \mid \begin{array}{l}
k \\
k
\end{array}\right) \cdot \Delta Y=\Delta\left(R \mid \begin{array}{l}
k \\
k
\end{array}\right) \cdot \Delta X \cdot \Delta Y \\
& =\Delta\left(R \mid \begin{array}{l}
k \\
k
\end{array}\right) \cdot \Delta A \cdot \Delta B .
\end{aligned}
$$

This proves the second statement.

Part 2.

$$
\int_{G_{n}} \Delta[(R: A) \ominus B] d \phi_{n} R=\beta(n, k) \cdot \Delta A \cdot \Delta(B) .
$$

Proof. Use Part 1, the fact that $\phi_{n}$ is the Haar measure of $G_{n}$ (see 3.2), again Part 1, and Definition 3.1 to check

$$
\begin{aligned}
\int_{G_{n}} \Delta[(R: A) \ominus B] d \phi_{n} R & =\int_{G_{n}} \Delta[(V: R: A) \ominus(V: B)] d \phi_{n} R \\
& =\int_{G_{n}} \Delta[(R: A) \ominus(V: B)] d \phi_{n} R \\
& =\int_{G_{n}} \Delta[(R: U: A) \ominus(V: B)] d \phi_{n} R \\
& =\int_{G_{n}} \Delta\left(R \mid{ }_{k}^{k}\right) \cdot \Delta A \cdot \Delta B d \phi_{n} R=\beta(n, k) \cdot \Delta A \cdot \Delta \cdot B .
\end{aligned}
$$

The proof is complete.

4.2. THEOREM. If $f$ is countably Lipschitzian on the $\mathcal{L}_{k^{k}}$ measurable subset $U$ of $E_{k}$ to $E_{n}$, and $g$ is countably Lipschitzian on the $\mathcal{L}_{n-k^{n-k}}$ measurable subset $V$ of $E_{n-k}$ to $E_{n}$, then

$$
\begin{aligned}
\int_{G_{n}} \int_{E_{n}} \Omega\left[f, U ;\left(R: T_{z}: g\right), V\right] d \mathcal{L}_{n}^{n} z d \phi_{n} R \\
=\beta(n, k) \cdot \int_{U} J f(x) d \mathcal{L}_{k}^{k} x \cdot \int_{\nabla} J g(y) d \mathcal{L}_{n-k}^{n-k} y \\
=\beta(n, k) \cdot \int_{E_{n}} N(f, U, z) d \mathcal{L}_{n}^{k} z \cdot \int_{E_{n}} N(g, V, z) d \mathcal{L}_{n}^{n-k} z .
\end{aligned}
$$

Proof. Let $W$ be the set of all points of the form $(x \circ y)$ with $x \in U$ and $y \in V$. Then $W$ is an $\mathcal{L}{ }^{n}$ measurable subset of $E_{n}$.

The remainder of our argument is divided into four parts.

Part 1. If $R \in G_{n}$ and $z \in E_{n}$, then 


$$
\Omega\left[(R: f), U ;\left(T_{z}: g\right), V\right]=N\{[(R: f) \ominus g], W, z\} .
$$

Proof. To say that a pair $(x, y)$ has the properties $x \in U, y \in V,(R: f)(x)$ $=\left(T_{s}: g\right)(y)$ is equivalent to the statement $(x \circ y) \in W,(R: f)(x)=z+g(y)$, which is in turn equivalent to $(x \circ y) \in W,[(R: f) \ominus g](x \circ y)=z$.

This makes Part 1 evident.

Part 2. If $R \in G_{n}$, then $[(R: f) \ominus g]$ is countably Lipschitzian on $W$.

Part 3.

$$
\int_{G_{n}} J[(R: f) \ominus g](x \circ y) d \phi_{n} R=\beta(n, k) \cdot J f(x) \cdot J f(y)
$$

for $\mathcal{L}_{n}{ }^{n}$ almost all $(x \circ y)$ in $W$.

Proof. For almost all $(x \circ y)$ in $W$ we know that $f$ is approximately differentiable at $x$, and $g$ is approximately differentiable at $y$.

Now pick such a point $(x \circ y)$.

Let $A$ be the approximate differential of $f$ at $x$, and let $B$ be the approximate differential of $g$ at $y$. Then $A \in M_{n}{ }^{k}$ and $B \in M_{n}{ }^{n-k}$.

Furthermore, if $R \in G_{n}$, then $(R: A)$ is the approximate differential of $(R: f)$ at $x$; hence $[(R: A) \ominus B]$ is the approximate differential of $[(R: f) \ominus g]$ at $(x \circ y)$.

Together with Lemma 4.1 this implies

$$
\begin{aligned}
\beta(n, k) \cdot J f(x) \cdot J f(y) & =\beta(n, k) \cdot \Delta A \cdot \Delta B \\
& =\int_{G_{n}} \Delta[(R: A) \ominus B] d \phi_{n} R=\int_{G_{n}} J[(R: f) \ominus g](x \circ y) d \phi_{n} R .
\end{aligned}
$$

Part 4.

$$
\begin{aligned}
\int_{G_{n}} \int_{E_{n}} \Omega\left[f, U ;\left(R: T_{z}: g\right), V\right] d \mathcal{L}_{n}^{n} z d \phi_{n} R & \\
& =\beta(n, k) \cdot \int_{U} J f(x) d \mathcal{L}_{k}^{k} x \cdot \int_{\nabla} J g(y) d \mathcal{L}_{n-k}^{n-k} y .
\end{aligned}
$$

Proof. First we use the Fubini Theorem and Part 3 to infer

$$
\begin{aligned}
\beta(n, k) \cdot \int_{U} J f(x) d \mathcal{L}_{k}^{k} x \cdot \int_{V} J g(y) & d \mathcal{L}_{n-k}^{n-k} y \\
& =\int_{W} \beta(n, k) \cdot J f(x) \cdot J g(y) d \mathcal{L}_{n}^{n}(x \circ y) \\
& =\int_{W} \int_{G_{n}} J[(R: f) \ominus g](x \circ y) d \phi_{n} R d \mathcal{L}_{n}^{n}(x \circ y) \\
& =\int_{W} \int_{G_{n}} J[(R: f) \ominus g](w) d \phi_{n} R d \mathcal{L}_{n}^{n} w .
\end{aligned}
$$

Next, to justify interchange of the order of integration, we must know 
that $J f[(R ; f) \ominus g](w)$ is measurable in $(R, w)$ with respect to the product measure of $\phi_{n}$ and $\mathcal{L}_{n}{ }^{n}$ over the cartesian product of $G_{n}$ and $W$. In fact $J[(R ; f) \ominus g](x \circ y)$ is the absolute value of the determinant of the differential of $[(R: f) \Theta g]$ at $(x \circ y)$. Applying Laplace's development to the first $k$ columns, we can express this determinant as a sum of products whose factors contain only $(R, x)$ or $y$ respectively. Now each factor of the first kind is in turn a determinant which can, according to the Cauchy-Binet theorem on the multiplication of matrices, be expressed as a sum of products whose factors contain only $R$ or $x$ respectively. Thus $J[(R: f) \ominus g](x \circ y)$ can be expressed as the absolute value of a finite sum of products whose factors are $\phi_{n}$ measurable in $R$ or $\mathcal{L}_{n}{ }^{n}$ measurable in $(x \circ y)$ respectively.

Thus Fubini's theorem applies. Letting $R^{\prime}$ be the matrix inverse to $R$, we use Part 2, [F2, 5.1], Part 1, and the defining properties of $\phi_{n}$ (see 3.2) to obtain:

$$
\begin{aligned}
\int_{G_{n}} \int_{W} J[(R: f) \ominus g](w) d \mathcal{L}_{n}^{n} w d \phi_{n} R \\
=\int_{G_{n}} \int_{E_{n}} N\{[(R: f) \ominus g] ; W, z\} d \mathcal{L}_{n}^{n} z d \phi_{n} R \\
=\int_{G_{n}} \int_{E_{n}} \Omega\left[(R: f), U ;\left(T_{2}: g\right), V\right] d \mathcal{L}_{n}^{n} z d \phi_{n} R \\
=\int_{G_{n}} \int_{E_{n}} \Omega\left[\left(R^{\prime}: f\right), U ;\left(T_{z}: g\right), V\right] d \mathcal{L}_{n}^{n} z d \phi_{n} R \\
=\int_{G_{n}} \int_{E_{n}} \Omega\left[f, U ;\left(R: T_{z}: g\right), V\right] d \mathcal{L}_{n}^{n} z d \phi_{n} R .
\end{aligned}
$$

This completes the proof of Part 4; from Part 4 and [F2, 5.1] the theorem follows at once.

4.3. REMARK. Suppose $f$ is a Lipschitzian function on $E_{k}$ to $E_{n}$ and $g$ is a Lipschitzian function on $E_{n-k}$ to $E_{n}$. Prompted by Theorem 4.2, and Part 1 of its proof, we introduce, for each set $X \subset E_{n}$, the function $t_{X}$ on the cartesian product $\left(E_{n} \times G_{n}\right)$ by the relation

$$
t_{X}(z, R)=N\{[(R: f) \ominus g], X, z\} \quad \text { for }(z, R) \in\left(E_{n} \times G_{n}\right),
$$

and investigate the measurability of the function $t_{X}$ with respect to the product measure, $\psi_{n}$, of $\mathcal{L}_{n}{ }^{n}$ and $\phi_{n}$.

Statement 1. If $X$ is an $F_{\sigma}$ subset of $E_{n}$, then $t_{X}$ is a $\psi_{n}$ measurable function.

Proof. Let $h$ be the function on $\left(E_{n} \times G_{n}\right)$ to $\left(E_{n} \times G_{n}\right)$ such that

$$
h(z, R)=([(R: f) \ominus g](z), R) \in\left(E_{n} \times G_{n}\right) \quad \text { for } \quad(z, R) \in\left(E_{n} \times G_{n}\right),
$$

and check that 


$$
t_{X}(z, R)=N\left[h,\left(X \times G_{n}\right),(z, R)\right] \quad \text { for }(z, R) \in\left(E_{n} \times G_{n}\right) \text {. }
$$

Now let $F$ be the family of all bounded $F_{\sigma}$ subsets of $\left(E_{n} \times G_{n}\right)$, recall that $h$ is continuous, hence

$$
S \in F \text { implies } h^{*}(S) \in F,
$$

and that the members of $F$ are $\psi_{n}$ measurable sets. Accordingly we obtain the relation

$$
0 \leqq \int N\left\{h,\left(X \times G_{n}\right),(z, R)\right\} d \psi_{n}(z, R)
$$

from $[F 1,4.1]$. This proves Statement 1.

Statement 2. If $X$ is an $\mathcal{L}_{n}{ }^{n}$ measurable subset of $E_{n}$, then $t_{x}$ is a $\psi_{n}$ measurable function.

Proof. Since $f$ and $g$ are Lipschitzian there is a number $A$ such that

$$
J[(R: f) \ominus g](w) \leqq A
$$

for $\mathcal{L}_{n}{ }^{n}$ almost all $w$ in $E_{n}$, and every $R \in G_{n}$.

If $C$ and $U$ are arbitrary sets such that

$$
C \subset X \subset U \subset E_{n}, C \text { is closed, } U \text { is open, }
$$

then

$$
t_{C}(z, R) \leqq t_{X}(z, R) \leqq t_{U}(z, R) \quad \text { for } \quad(z, R) \in\left(E_{n} \times G_{n}\right) ;
$$

moreover Statement 1 and $[F 2,4.5]$ imply

$$
\begin{aligned}
\int\left\{t_{U}(z, R)-t_{F}(z, R)\right\} d \psi_{n}(z, R) \\
=\int t_{(U-F)}(z, R) d \psi_{n}(z, R) \\
\quad=\int_{G_{n}} \int_{E_{n}} N\{[(R: f) \ominus g],(U-F), z\} d \mathcal{L}_{n}^{n}{ }_{n} z d \phi_{n} R \\
=\int_{G_{n}} \int_{(U-F)} J[(R: f) \ominus g](w) d \mathcal{L}_{n}^{n} w d \phi_{n} R \leqq A \cdot \mathcal{L}_{n}^{n}(U-F),
\end{aligned}
$$

that is

$$
\int\left\{t_{U}(R, z)-t_{F}(R, z)\right\} d \psi_{n}(z, R) \leqq A \cdot \mathcal{L}_{n}^{n}(U-F) .
$$

Now $\mathcal{L}_{n}{ }^{n}(U-F)$ can be made arbitrarily small by suitable choices of $C$ and $U$. Accordingly $t_{x}$ can be approximated from below and from above by $\psi_{n}$ measurable functions, $t_{c}$ and $t_{U}$, whose integrals differ by as little as we please. Hence $t_{X}$ is a $\psi_{n}$ measurable function. 
These results are obviously still true if $f$ and $g$ are only countably Lipschitzian.

4.4. Lemma. If $A \in M_{n}^{k}$, then

$$
\int_{G_{n}} \Delta\left(P_{R}^{k}: A\right) d \phi_{n} R=\beta(n, k) \cdot \Delta A .
$$

Proof. For $R \in G_{n}$ and $i=1,2, \cdots, k ; j=1,2, \cdots, k$, we have

$$
\left(P_{R}^{k}: A\right)_{i}^{j}=\left[\left(\left.R\right|_{k} ^{n}\right): A\right]_{i}^{j}=\sum_{m=1}^{n}\left(\left.R\right|_{k} ^{n}\right)_{i}^{m} A_{m}^{j}=\sum_{m=1}^{n} R_{i}^{m} A_{m}^{j}=(R: A)_{i}^{j} .
$$

Hence $\left(P_{R^{k}}: A\right)=\left[(R: A) \mid k_{k}^{k}\right]$ for $R \in G_{n}$.

Now choose $B \in M_{n}{ }^{n-k}$ as follows: Let $j=1,2, \cdots, n-k$; let $i=1$, $2, \cdots, n$; and put $B_{j+k}{ }^{i}=1, B_{i}{ }^{i}=0$ for $i \neq j+k$.

Next we check

$$
\Delta[(R: A) \ominus B]=\Delta\left[\left.(R: A)\right|_{k} ^{k}\right]=\Delta\left(P_{R}^{k}: A\right) \quad \text { for } \quad R \in G_{n},
$$

and apply Lemma 4.1 to conclude

$$
\begin{aligned}
\int_{G_{n}} \Delta\left(P_{R}^{k}: A\right) d \phi_{n} R & =\int_{G_{n}} \Delta[(R: A) \ominus B] d \phi_{n} R \\
& =\beta(n, k) \cdot \Delta A \cdot \Delta B=\beta(n, k) \cdot \Delta A .
\end{aligned}
$$

4.5. TheOREM. If $f$ is countably Lipschitzian on the $\mathcal{L}_{k^{k}}{ }^{k}$ measurable subset $U$ of $E_{k}$ to $E_{n}$, then

$$
\begin{aligned}
\int_{G_{n}} \int_{E_{k}} N\left[\left(P_{R}^{k}: f\right), U, x\right] d \mathcal{L}_{k}^{k} x d \phi_{n} R & =\beta(n, k) \cdot \int_{U} J f(x) d \mathcal{L}_{k}^{k} x \\
& =\beta(n, k) \cdot \int_{E_{n}} N(f, U, z) d \mathcal{L}_{n z}^{k} .
\end{aligned}
$$

Proof. If $R \in G_{n}$, then $\left(P_{R}^{k}: f\right)$ is countably Lipschitzian on $U$ to $E_{k}$; and if $A$ is the approximate differential of $f$ at a point $x \in U$, then $\left(P_{R^{k}}: A\right)$ is the approximate differential of $\left(P_{R^{k}}: f\right)$ at that point $x$.

Hence Lemma 4.4 implies

$$
\int_{G_{n}} J\left(P_{R}^{k}: f\right)(x) d \phi_{n} R=\beta(n, k) \cdot J f(x)
$$

for $\mathcal{L}_{k}{ }^{k}$ almost all $x$ in $U$.

To complete the proof we integrate over $U$ (with respect to the measure $\mathcal{L}_{k}{ }^{k}$ ), interchange the order of integration (which can be justified as in 4.2), and apply $[\mathrm{F} 2,5.1]$. 
4.6. TheoRem. If $A \subset E_{n}$ and there are $\mathcal{L}_{n}{ }^{k}$ measurable subsets $U_{1}, U_{2}$, $U_{3}, \cdots$ of $E_{k}$ and Lipschitzian functions $f_{1}, f_{2}, f_{3}, \cdots$ on $E_{k}$ to $E_{n}$ such that

$$
\sum_{i=1}^{\infty} f_{i}^{*}\left(U_{i}\right) \subset A, \quad \mathcal{L}_{n}^{k}\left\{A-\sum_{i=1}^{\infty} f_{i}^{*}\left(U_{i}\right)\right\}=0,
$$

then

$$
\int_{G_{n}} \int_{E_{k}} N\left(P_{R}^{k}, A, x\right) d \mathcal{L}_{k}^{k} x d \phi_{n} R=\beta(n, k) \cdot \mathcal{L}_{n}^{k}(A) .
$$

Proof. Let $A_{i}=f_{i}^{*}\left(U_{i}\right)$ for $i=1,2,3, \cdots$,

$$
X=A-\sum_{i=1}^{\infty} A_{i}
$$

In view of a known method of reduction (see $[\mathrm{MF}]$ ), we assume that

$$
A_{i} A_{j}=0 \quad \text { for } i \neq j,
$$

and $f_{i}$ is univalent on $U_{i}$ for each positive integer $i$.

Hence $[F 2,4.5,2.3]$ and Theorem 4.4 of the present paper enable us to compute:

$$
\begin{aligned}
\beta(n, k) \mathcal{L}_{n}^{k}(A)= & \beta(n, k) \cdot\left\{\mathcal{L}_{n}^{k}(X)+\sum_{i=1}^{\infty} \mathcal{L}_{n}^{k}\left(A_{i}\right)\right\} \\
= & \beta(n, k) \sum_{i=1}^{\infty} \int_{E_{n}} N\left(f_{i}, U_{i}, x\right) d \mathcal{L}_{n}^{k} x \\
= & \sum_{i=1}^{\infty} \int_{G_{n}} \int_{E_{k}} N\left[\left(P_{R}^{k}: f_{i}\right), U_{i}, z\right] d \mathcal{L}_{k}^{k} z d \phi_{n} R \\
= & \int_{G_{n}} \int_{E_{k}} N\left(P_{R}^{k}, X, z\right) d \mathcal{L}_{k}^{k} z d \phi_{n} R \\
& +\sum_{i=1}^{\infty} \int_{G_{n}} \int_{E_{k}} N\left(P_{R}^{k}, A_{i}, z\right) d \mathcal{L}_{k z}^{k} z \phi_{n} R \\
= & \int_{G_{n}} \int_{E_{k}}\left\{N\left(P_{R}^{k}, X, z\right)+\sum_{i=1}^{\infty} N\left(P_{R}^{k}, A_{i}, z\right)\right\} d \mathcal{L}_{k}^{k} z d \phi_{n} R \\
= & \int_{G_{n}} \int_{E_{k}} N\left(P_{R}^{k}, A, z\right) d \mathcal{L}_{k}^{k} z d \phi_{n} R .
\end{aligned}
$$

4.7. REMARK. It would be highly interesting to know whether hypothesis and conclusion of Theorem 4.6 are equivalent under the assumption that $\mathcal{L}^{k}(A)<\infty$. 
In the special case $n=2, k=1$, this question has been answered in the affirmative by Sherman, who used Besicovitch's result that an irregular plane set of finite Caráthéodory linear measure projects in almost all directions into linear sets of Lebesgue measure zero. (See [BE], [SH ].)

The methods of Besicovitch, and of Morse and Randolph (see [MR]), yield certain generalizations to the case $n \geqq 2, k=n-1$, of their results in the case $n=2, k=1$. In fact if $\mathcal{L}_{n}{ }^{n-1}$ is replaced by $(n-1)$-dimensional Hausdorff measure, the corresponding question can be answered in the affirmative. The more difficult problem for $\mathcal{L}_{n}{ }^{n-1}$ is still unsolved.

We recall that $m$-dimensional Hausdorff measure, $\mathcal{F C}_{n}{ }^{m}$, is defined by a procedure analogous to that defining $\mathcal{L}_{n}{ }^{m}$ (see $[F 2,2.1]$, where $\mathcal{L}_{n}{ }^{m}$ is called $\Phi$ ). The only changes are that the function $\gamma$, such that

$$
\gamma(S)=\sup _{R \in G_{n}} \mathcal{L}_{m}^{m}\left[P_{R}^{m *}(S)\right] \quad \text { for } S \subset E_{n},
$$

which occurs in the definition of $\mathcal{L}_{n}{ }^{m}$, is replaced by the function $\gamma^{\prime}$, such that

$$
\gamma^{\prime}(S)=\alpha(m) \cdot 2^{-m} \cdot(\operatorname{diam} S)^{m} \quad \text { for } S \subset E_{n},
$$

and that the open sets occurring in the coverings are no longer required to be connected.

If $X \subset E_{n}$, the relations

$$
\begin{aligned}
& \mathcal{L}_{n}^{m}(X) \leqq \mathcal{F}_{n}^{m}(X) \quad \text { for } m=1,2,3, \cdots, n, \\
& \mathcal{L}_{n}^{1}(X)=\mathcal{F C}_{n}^{1}(X), \\
& \mathcal{L}_{n}^{n}(X)=\mathcal{F C}_{n}^{n}(X)
\end{aligned}
$$

follow without difficulty from the definitions and the fact that, of all subsets of $E_{m}$ with a given diameter, the sphere has the largest $\mathcal{L}_{m}{ }^{m}$ measure.

4.8. Remark. The results mentioned in 4.7 lead to the following theorem of Gauss-Green type:

If $A$ is a bounded open subset of $E_{n}, B$ is the boundary of $A, \mathcal{F}_{n}{ }^{n-1}(B)<\infty$, and the numerically valued function $f$ is differentiable on $A$, continuous on $(A+B)$, and such that

then

$$
\int_{A}\left|D_{j} f(x)\right| d \mathcal{L}_{n}^{n} x<\infty \quad \text { for } j=1,2, \cdots, n,
$$

$$
\int_{A} D_{j} f(x) d \mathcal{L}_{n}^{n} x=\int f(x) \nu_{j}(A, x) d \mathcal{F}_{n}^{n-1} x \quad \text { for } j=1,2, \cdots, n .
$$

Here $\nu_{j}(A, x)$ is the $j$ th component of the exterior normal of $A$ at $x$ (defined in $[\mathrm{F} 3,3.6])$, and $D_{i} f$ is the partial derivative of $f$ in the direction of the $j$ th unit vector. 
In fact the methods and results of our recent paper on the Gauss-Green Theorem, [F3], remain valid when $\mathcal{L}_{n}{ }^{n-1}$ (which was there called $\Phi$ ) is replaced by $\mathcal{F C}_{n}{ }^{n-1}$. Further, if $Q$ is the set of those points of $B$ at which $B$ is $\mathcal{H C}_{n}{ }^{n-1}$ restricted (see $[\mathrm{F} 3,3.12]$ ), it can be shown by the methods mentioned in 4.7 that

$$
\mathcal{L}_{n-1}^{n-1}\left[P_{R}^{n-1 *}(B-Q)\right]=0 \quad \text { for } \phi_{n} \text { almost all } R \text { in } G_{n} .
$$

In virtue of the well known correspondence of the projecting functions, $P_{R^{n-1}}$, and the points of the unit sphere

$$
S=E_{n} \underset{z}{E}[|z|=1],
$$

we infer from the modified $\left(\mathcal{F C}_{n}{ }^{n-1}\right.$ replaces $\left.\mathcal{L}_{n}{ }^{n-1}=\Phi\right)$ theorem $[\mathrm{F} 3,6.4]$ that

$$
\int_{A}[\operatorname{grad} f(x) \cdot z] d \mathcal{L}_{n}^{n} x=\int f(x)[\nu(A, x) \cdot z] d \mathcal{C}_{n}^{n-1} x
$$

for $\mathcal{L}_{n}{ }^{n-1}$ almost all $z$ in $S$. Accordingly

$$
\left\{\int_{A} \operatorname{grad} f(x) d \mathcal{L}_{n}^{n} x-\int f(x) \nu(A, x) d \mathcal{F}_{n}^{n-1} x\right\} \cdot z=0
$$

for $\mathcal{L}_{n}{ }^{n-1}$ almost all $z$ in $S$, which implies

$$
\int_{A} \operatorname{grad} f(x) d \mathcal{L}_{n}^{n} x-\int f(x) \nu(A, x) d \mathcal{H C}_{n}^{n-1} x=\theta,
$$

the origin of $E_{n}$. This is equivalent to the relation

$$
\int_{A} D_{j} f(x) d \mathcal{L}_{n}^{n} x=\int f(x) \nu_{j}(A, x) d \mathcal{C}_{n}^{n-1} x \quad \text { for } j=1,2, \cdots, n .
$$

The assumption that $f$ be differentiable on $A$ is unnecessarily restrictive. It is sufficient to assume that $f$ be absolutely continuous in the sense of Tonelli on $A$.

5. Determination of $\beta(n, k)$. In this section we evaluate the definite integral $\beta(n, k)$ over the group $G_{n}$ in terms of the function $\Gamma$.

5.1. Lemma. If $n$ is an integer not less than 2, then

Proof. Let

$$
\beta(n, 1)=\frac{\alpha(1) \cdot \alpha(n-1)}{n \cdot \alpha(n)} .
$$

$$
A=E_{n} \underset{z}{E}[|z|=1], \quad B=E_{n-1} \underset{z}{E}[|x| \leqq 1],
$$

and let $I$ be the identity of $G_{n}$. Then $R \in G_{n}$ implies 


$$
\begin{array}{r}
N\left(P_{R}^{n-1}, A, x\right)=N\left[\left(P_{I}^{n-1}: R\right), A, x\right]=N\left[P_{I}^{n-1}, R^{*}(A), x\right]=N\left[P_{I}^{n-1}, A, x\right] \\
=2 \text { for }|x|<1, \text { and }=0 \text { for }|x|>1 .
\end{array}
$$

Hence Theorem 4.6 enables us to conclude

$$
\begin{aligned}
\beta(n, n-1) \mathcal{L}_{n}^{n-1}(A) & =\int_{G_{n}} 2 \mathcal{L}_{n-1}^{n-1}(B) d \phi_{n} R, \\
\beta(n, n-1) \cdot n \alpha(n) & =2 \alpha(n-1)=\alpha(1) \cdot \alpha(n-1) .
\end{aligned}
$$

Recall that $\beta(n, k)=\beta(n, n-k)$ to complete the proof.

5.2. Lemma. If $a \in E_{n}$ and $b \in E_{n}$, then

$$
\int_{G_{n}}|a \cdot R(b)| d \phi_{n} R=|a| \cdot|b| \cdot \beta(n, 1) .
$$

Proof. Choose $U \in G_{n}$ and $V \in G_{n}$ such that

$$
a=|a| U^{1}, \quad b=|b| V^{1} .
$$

Denote the conjugate (transpose) of any element $R \in G_{n}$ by $\bar{R}$, and remember that $\bar{R}$ is characterized by the property

$$
x \cdot R(y)=\bar{R}(x) \cdot y \quad \text { for } \quad x \in E_{n}, y \in E_{n} .
$$

Further recall that $\bar{R}$ is the inverse of $R$ for each orthogonal matrix $R$. Hence

$$
\begin{aligned}
\int_{G_{n}}|a \cdot R(b)| d \phi_{n} R & =\int_{G_{n}}\left|a \cdot R\left(|b| V^{1}\right)\right| d \phi_{n} R \\
& =|b| \int_{G_{n}}\left|a \cdot(R: V)^{1}\right| d \phi_{n} R=|b| \int_{G_{n}}\left|a \cdot R^{1}\right| d \phi_{n} R \\
& =|b| \int_{G_{n}}\left|\bar{R}(a) \cdot I^{1}\right| d \phi_{n} R \\
& =|a| \cdot|b| \int_{G_{n}}\left|\bar{R}\left(U^{1}\right) \cdot I^{1}\right| d \phi_{n} R \\
& =|a| \cdot|b| \int_{G_{n}}\left|(\bar{R}: U)^{1} \cdot I^{1}\right| d \phi_{n} R \\
& =|a|:|b| \int_{G_{n}}\left|R^{1}, I^{1}\right| d \phi_{n} R \\
& =|a| \cdot|b| \int_{G_{n}}\left|R_{1}^{1}\right| d \phi_{n} R=|a| \cdot|b| \cdot \beta(n, 1) .
\end{aligned}
$$


5.3. Lemma. If $k<n$ are positive integers, then

$$
\beta(n, k) \cdot \beta(k, 1)=\beta(n, k-1) \cdot \beta(n-k+1,1) .
$$

Proof. Let $I$ be the unit matrix in $M_{n}{ }^{n}$. For each matrix $S \in M_{n-k+1}^{n-k+1}$ we define ${ }^{*} S \in M_{n}{ }^{n}$ by the relations

$$
\begin{array}{ll}
{ }^{*} S_{i}^{j}=I_{i}^{j} & \text { if } i<k \text { or } j<k, \\
{ }^{*} S_{i}^{j}=S_{i-k+1}^{j-k+1} & \text { if } i \geqq k \text { and } j \geqq k .
\end{array}
$$

Then $S \in G_{n-k+1}$ implies $* S \in G_{n}$ and

$$
\beta(n, k)=\int_{G_{n}} \Delta\left(\left.R\right|_{k} ^{k}\right) d \phi_{n} R=\int_{G_{n}} \Delta\left[\left.\left(R:{ }^{*} S\right)\right|_{k} ^{k}\right] d \phi_{n} R .
$$

Hence

$$
\begin{aligned}
\beta(n, k) & =\int_{G_{n-k+1}} \int_{G_{n}} \Delta\left[\left.(R: * S)\right|_{k} ^{k}\right] d \phi_{n} R d \phi_{n-k+1} S \\
& =\int_{G_{n}} \int_{G_{n-k+1}} \Delta\left[\left.(R: * S)\right|_{k} ^{k}\right] d \phi_{n-k+1} S d \phi_{n} R .
\end{aligned}
$$

The lemma is a consequence of this formula, and of the two parts into which we divide the remainder of the argument.

Part 1. If $R \in G_{n}$, then

$$
\int_{G_{n-k+1}} \Delta\left[\left.(R: * S)\right|_{k} ^{k}\right] d \phi_{n-k+1} S=\beta(n-k+1,1) \cdot \Delta\left(\left.R\right|_{k} ^{k-1}\right) .
$$

Proof. Define $f \in M_{n}^{n-k+1}$ by the relation

$$
f(x)=x_{1} R^{k}+x_{2} R^{k+1}+\cdots+x_{n-k+1} R^{n} \quad \text { for } \quad x \in E_{n-k+1},
$$

and observe that

$$
f(x) \cdot f(y)=x \cdot y \quad \text { for } \quad x \in E_{n-k+1}, y \in E_{n-k+1} .
$$

Furthermore $S \in M_{n-k+1}^{n-k+1}$ implies

and

$$
\begin{aligned}
\left(R:{ }^{*} S\right)^{k}= & R\left({ }^{*} S^{k}\right)=\sum_{j=1}^{n} * S_{j}^{k} R^{j}=S_{1}^{1} R^{k}+S_{2}^{1} R^{k+1}+\cdots+S_{n-k+1}^{1} R^{n}, \\
\left(R:{ }^{*} S\right)^{k} & =f\left(S^{1}\right),
\end{aligned}
$$

$$
\left(R:{ }^{*} S\right)^{i}=R\left({ }^{*} S^{j}\right)=R\left(I^{i}\right)=R^{i} \quad \text { for } j=1,2, \cdots, k-1 .
$$

How let $A_{i}$ be the $k-1$ rowed minor of $\left(\left.R\right|_{k} ^{k-1}\right)$ which is obtained by deleting the $i$ th row from $\left(\left.R\right|_{k} ^{k-1}\right)$. Pick $c \in E_{n}$ so that 


$$
\begin{aligned}
c_{i} & =(-1)^{i} \operatorname{det} A_{i} \\
c_{i} & =0
\end{aligned}
$$$$
\text { for } i=1,2, \cdots, k \text {; }
$$$$
\text { for } i=k+1, \cdots, n \text {. }
$$

Then

$$
c \cdot R^{j}=0 \quad \text { for } j=1,2, \cdots, k-1,
$$

because $c \cdot R^{j}$ equals the determinant of the matrix obtained from $\left(\left.R\right|_{k} ^{k}\right)$ by replacing the $k$ th column by the point

$$
\left(R_{1}^{j}, R_{2}^{j}, \cdots, R_{k}^{j}\right) \in E_{k},
$$

and the matrix so formed has two equal columns.

Hence a point $a \in E_{n-k+1}$ can be found for which $c=f(a)$.

From all this it follows that

$$
\begin{aligned}
\Delta\left[\left(R:{ }^{*} S\right) \mid \begin{array}{l}
k \\
k
\end{array}\right] & =\left|\sum_{i=1}^{k} c_{i}\left(R:{ }^{*} S\right)_{i}^{k}\right|=\left|\sum_{i=1}^{n} c_{i} R\left({ }^{*} S^{k}\right)_{i}\right| \\
& =\left|c \cdot R\left({ }^{*} S^{k}\right)\right|=\left|f(a) \cdot f\left(S^{1}\right)\right|=\left|a \cdot S^{1}\right|
\end{aligned}
$$

for every $S \in G_{n-k+1}$, and we use Lemma 5.2 to conclude

$$
\begin{aligned}
\int_{G_{n-k+1}} \Delta\left[(R: * S) \mid \begin{array}{l}
k \\
k
\end{array}\right] d \phi_{n-k+1} S & =\int_{G_{n-k+1}}\left|a \cdot S^{1}\right| d \phi_{n-k+1} S \\
& =|a| \cdot \beta(n-k+1,1)=|c| \cdot \beta(n-k+.1,1) \\
& =\Delta\left(\left.R\right|_{k} ^{k-1}\right) \cdot \beta(n-k+1,1) .
\end{aligned}
$$

Part 2. $\beta(k, 1) \cdot \int_{G_{n}} \Delta\left(\left.R\right|_{k^{k-1}}\right) d \phi_{n} R=\beta(n, k-1)$.

Proof. For each matrix $U \in M_{k}^{k}$ we define ${ }^{*} U \in M_{n}{ }^{n}$ by the relations

$$
\begin{array}{ll}
{ }^{*} U_{i}^{j}=U_{i}^{j} & \text { if } i \leqq k \text { and } j\rfloor \leqq k, \\
{ }^{*} U_{i}^{j}=I_{i}^{j} & \text { if } i>k \text { or } j>{ }^{j} k
\end{array}
$$

Then $U \in G_{k}$ implies ${ }^{*} U \in G_{n}$; and if $R \in G_{n}$ and $i=1,2, \cdots, k-1$; $j=1,2, \cdots, k-1$, we have

$$
\begin{aligned}
{\left[\left(\left.U\right|_{k-1} ^{k}\right):\left(\left.R\right|_{k} ^{k-1}\right)\right]_{i}^{j} } & =\sum_{m=1}^{k}\left(\left.U\right|_{k-1} ^{k}\right)_{i}^{m}\left(\left.R\right|_{k} ^{k-1}\right)_{m}^{j}=\sum_{m=1}^{k} U_{i}^{m} R_{m}^{j} \\
& =\sum_{m=1}^{n} * U_{i}^{m} R_{m}^{j}=\left({ }^{*} U: R\right)_{i}^{j}
\end{aligned}
$$

It follows that

$$
\left[\left(\left.U\right|_{k-1} ^{k}\right):\left(\left.R\right|_{k} ^{k-1}\right)\right]=\left[\left.\left({ }^{*} U: R\right)\right|_{k-1} ^{k-1}\right] \text { for } U \in G_{n-k+1}, R \in G_{n} .
$$


Use Lemma 4.4 to check

$$
\begin{aligned}
\beta(k, 1) \cdot \Delta\left(\left.R\right|_{k} ^{k-1}\right) & =\beta(k, k-1) \Delta\left(\left.R\right|_{k} ^{k-1}\right) \\
& =\int_{G_{k}} \Delta\left[\left(\left.U\right|_{k-1} ^{k}\right):\left(\left.R\right|_{k} ^{k-1}\right)\right] d \phi_{k} U \\
& =\int_{G_{k}} \Delta\left[\left.\left({ }^{*} U: R\right)\right|_{k-1} ^{k-1}\right] d \phi_{k} U
\end{aligned}
$$

for each $R \in G_{n}$.

Integrating over $G_{n}$ we obtain

$$
\begin{aligned}
\beta(k, 1) \int_{G_{n}} \Delta\left(\left.R\right|_{k} ^{k-1}\right) d \phi_{n} R & =\int_{G_{k}} \int_{G_{n}} \Delta\left[\left.\left({ }^{*} U: R\right)\right|_{k-1} ^{k-1}\right] d \phi_{n} R d \phi_{k} U \\
& =\int_{G_{k}} \int_{G_{n}} \Delta\left(\left.R\right|_{k-1} ^{k-1}\right) d \phi_{n} R d \phi_{k} U=\beta(n, k-1) .
\end{aligned}
$$

This completes the proof of Part 2, and of the lemma.

5.4. TheOREM. If $k<n$ are positive integers, then

$$
\beta(n, k)=\frac{\alpha(k) \cdot \alpha(n-k)}{\alpha(n) \cdot C_{n, k}}=\frac{\Gamma((k+1) / 2) \Gamma((n-k+1) / 2)}{\Gamma(1 / 2) \cdot \Gamma((n+1) / 2)} .
$$

Proof. Let $n$ be a positive integer. We use induction in $k$.

We know from Lemma 5.1 that

$$
\beta(n, 1)=\frac{\alpha(1) \cdot \alpha(n-1)}{\alpha(n) \cdot C_{n, 1}} .
$$

Now if $k$ is any positive integer less than $n$, and if

$$
\beta(n, k-1)=\frac{\alpha(k-1) \cdot \alpha(n-k+1)}{\alpha(n) \cdot C_{n, k-1}},
$$

then the Lemmas 5.3 and 5.1 imply

$$
\begin{aligned}
\beta(n, k) & =\beta(n, k-1) \cdot \frac{\beta(n-k+1,1)}{\beta(k, 1)} \\
& =\frac{\alpha(k-1) \cdot \alpha(n-k+1)}{\alpha(n) \cdot C_{n, k-1}} \cdot \frac{\alpha(1) \cdot \alpha(n-k)}{(n-k+1) \cdot \alpha(n-k+1)} \cdot \frac{k \cdot \alpha(k)}{\alpha(1) \cdot \alpha(k-1)} \\
& =\frac{\alpha(k) \cdot \alpha(n-k)}{\alpha(n) \cdot C_{n, k}} .
\end{aligned}
$$

This shows that the first equality in our theorem holds for all positive integers $k<n$. 
The second equality follows immediately from 2.5 .

6. Stable values and area.

6.1. Definition. Suppose $f$ is a continuous function on a subset $X$ of $E_{k}$ to $E_{n}$. Following [HW, p. 74], we say a point $y$ is a stable value of $f$ if and only if there is a number $\epsilon>0$ such that for each continuous function $g$ on $X$ to $E_{n}$, satisfying the relation

$$
|g(x)-f(x)|<\epsilon \quad \text { for } \quad x \in X,
$$

there exists a point $w \in X$ with $g(w)=y$.

6.2. Lemma. If $f$ is continuous on $X$ to $E_{n}$ and $y$ is a stable value of $f$, then there is a number $\epsilon>0$ such that

$$
z \text { is a stable value of } g \text {, }
$$

whenever

$$
|z-y|<\epsilon
$$

$g$ is continuous on $X$ to $E_{n}$,

$$
|g(x)-f(x)|<\epsilon \quad \text { for } x \in X .
$$

Proof. Pick $\delta>0$ so that for every continuous function $t$ on $X$ to $E_{n}$ the relation

$$
|t(x)-f(x)|<\delta \quad \text { for } \quad x \in X
$$

implies the existence of a point $w \in X$ for which $t(w)=y$.

Take $\epsilon=\delta / 3$.

Now suppose $g$ is continuous on $X$ to $E_{n}$ with

$$
|g(x)-f(x)|<\epsilon \quad \text { for } x \in X,
$$

and pick a point $z \in E_{n}$ for which

$$
|z-y|<\epsilon \text {. }
$$

To prove that $z$ is a stable value of $g$, we let $h$ be any continuous function on $X$ to $E_{n}$, with the property

$$
|h(x)-g(x)|<\epsilon \quad \text { for } \quad x \in X .
$$

Define the function $t$ by the relation

$$
t(x)=h(x)+y-z \quad \text { for } \quad x \in X
$$

and check that

$$
|t(x)-f(x)|<3 \epsilon=\delta \quad \text { for } \quad x \in X .
$$

Hence a point $w \in X$ exists for which $t(w)=y$ or, equivalently, $h(w)=z$.

The proof is complete. 
6.3. Lemma. If $f$ is continuous and univalent on the open subset $X$ of $E_{n}$ to $E_{n}$, and $y \in f^{*}(X)$, then $y$ is a stable value of $f$. that

Proof. Use Brouwer's Theorem (see [HW, p. 96]) to select $r>0$ such

$$
U=E_{n} \underset{u}{E}[|u-y| \leqq r] \subset f^{*}(X)
$$

From [HW, p. 75] we know that $y$ is a stable value of the identity mapping of $U$. Accordingly there is a number $\epsilon>0$ such that for each continuous function $t$ on $U$ to $E_{n}$, satisfying the relation

$$
|t(u)-u|<\epsilon \quad \text { for } u \in U,
$$

there exists a point $w \in U$ with $t(w)=y$.

Now suppose $g$ is a continuous function on $X$ to $E_{n}$ such that

$$
|g(x)-f(x)|<\epsilon \quad \text { for } x \in X .
$$

Let $h$ be the inverse of $f$ and define

$$
t(u)=g[h(u)] \quad \text { for } u \in U .
$$

Check that $u \in U$ implies

$$
|t(u)-u|=|g[h(u)]-f[h(u)]|<\epsilon .
$$

Hence there is a point $w \in U$ for which $t(w)=y$; consequently

$$
h(w) \in X, \quad g[h(w)]=t(w)=y .
$$

6.4. THEOREM. If $f$ is a continuous function on a subset of $E_{n}$ to $E_{n}$, and $f$ has a nonsingular differential at a point $x \in E_{n}$, then $f(x)$ is a stable value of $f$.

Proof. Assume $x=f(x)=\theta$, the origin of $E_{n}$. Let

$$
Z=E_{n} \underset{z}{E}[|z|<1], \quad f_{r}(z)=\frac{f(r z)}{r} \quad \text { for } \quad r>0, z \in Z .
$$

For sufficiently small positive $r$, the function $f_{r}$ is continuous on $Z$ to $E_{n}$. In order to prove the theorem it will evidently suffice to show the existence of a number $r$ such that $\theta$ is a stable value of $f_{r}$.

Let $L$ be the differential of $f$ at $\theta$. Accordingly

$$
\frac{|f(u)-L(u)|}{|u|} \rightarrow 0 \text { as } u \rightarrow \theta .
$$

However

$$
\left|f_{r}(z)-L(z)\right|=\frac{|f(r z)-L(r z)|}{r} \leqq \frac{|f(r z)-L(r z)|}{|r z|} \quad \text { for } \quad r>0, z \in Z,
$$


so that

$$
f_{r}(z) \rightarrow L(z), \quad \text { uniformly for } z \in Z \text {, as } r \rightarrow 0 .
$$

Since $L$ is nonsingular we infer from Lemma 6.3 that $\theta$ is a stable value of $L$. We combine this fact with Lemma 6.2, and the just established convergence of $f_{r}$ to $L$, to infer that $\theta$ is a stable value of $f_{r}$ for all sufficiently small positive numbers $r$.

This completes our argument.

6.5. Definition. If $f$ is a function and $A$ is a set, then

is the function such that

$$
(f \mid A)
$$

$$
\begin{aligned}
\text { domain }(f \mid A) & =A \cdot \operatorname{domain} f, \\
(f \mid A)(x) & =f(x) \quad \text { for } \quad x \in(A \cdot \operatorname{domain} f) .
\end{aligned}
$$

6.6. Definition. Suppose $f$ is a function on a subset of $E_{k}$ to $E_{n}$. We say $f$ is stable at $x$ if and only if there is such an open set $U$ that $x \in U \subset \operatorname{domain} f$ and $f(x)$ is a stable value of $(f \mid V)$ whenever $V$ is an open set with $x \in V \subset U$.

6.7. Definition. If $f$ is a continuous function on a subset of $E_{k}$ to $E_{k}$, and if $y \in E_{k}$, then

$$
S(f, y)
$$

is the supremum (possibly 0 or $\infty$ ) of the set of all nonnegative integers $m$ such that there are nonvacuous open sets

$$
U_{1}, U_{2}, \cdots, U_{m},
$$

whose closures are disjoint compact subsets of the interior of the domain of $f$, and for which

$$
y \text { is a stable value of }\left(f \mid U_{i}\right) \quad \text { for } i=1,2, \cdots, m \text {. }
$$

6.8. Remark. Suppose $X \subset E_{k}$ and let $F$ be the family of all continuous functions on $X$ to $E_{n}$. Define convergence of a sequence of functions in $F$ to mean uniform convergence on every compact subset of the interior of $X$.

Then $S$ is lower semicontinuous on the cartesian product space $\left(F \times E_{n}\right)$.

This is a consequence of 6.7 and 6.2.

Further we note that, for each function $f \in F$ and each point $y \in E_{n}$, the number (possibly $\infty$ ) of points $x$ in the interior of $X$, at which $f$ is stable and $f(x)=y$, is less than or equal to $S(f, y)$.

6.9. Definition. For each continuous function $f$ whose domain is a subset of $E_{k}$ and whose range is a subset of $E_{n}$, with $k \leqq n$, we define

$$
\mathcal{A}(f)=\frac{1}{\beta(n, k)} \int_{G_{n}} \int_{E_{k}} S\left[\left(P_{R}^{k}: f\right), y\right] d \mathcal{L}_{k}^{k} y d \phi_{n} R .
$$

Note that the integrand is lower semicontinuous in virtue of Remark 6.8; 
hence the double integral exists, finite or infinite.

6.10. REMARK. If $k=n$, then

$$
\mathcal{A}(f)=\int_{E_{k}} S(f, y) d \mathcal{L}_{k}^{k} y .
$$

In fact $P_{R}{ }^{k}=R, S[(R: f), y]=S\left[f, R^{\prime}(y)\right]$ and $J R(y)=1$ whenever $R \in G_{k}$, $y \in E_{k}$. (Here $R^{\prime}$ is the inverse of $R$.) Consequently

$$
\begin{aligned}
\mathscr{A}(f) & =\int_{G_{k}} \int_{E_{k}} \delta\left[f, R^{\prime}(y)\right] d \mathcal{L}_{k}^{k} y d \phi_{k} R=\int_{G_{k}} \int_{E_{k}} \delta[f, R(y)] d \mathcal{L}_{k}^{k} y d \phi_{k} R \\
& =\int_{G_{k}} \int_{E_{k}} \delta[f, R(y)] J R(y) d \mathcal{L}_{k}^{k} y d \phi_{k} R=\int_{G_{k}} \int_{E_{k}} \delta(f, y) d \mathcal{L}_{k}^{k} y d \phi_{k} R \\
& =\int_{E_{k}} \delta(f, y) d \mathcal{L}_{k}^{k} y .
\end{aligned}
$$

6.11. THEOREM. If $k \leqq n$ are positive integers, $X \subset E_{k}$, the functions $f, g_{1}, g_{2}$, $g_{3}, \cdots$ are continuous with domain $X$ to $E_{n}$, and if

$$
g_{i}(x) \rightarrow f(x) \text {, uniformly for } x \in C \text {, as } i \rightarrow \infty,
$$

whenever $C$ is a compact subset of the interior of $X$, then

$$
\lim _{i \rightarrow \infty} \inf A\left(g_{i}\right) \geqq \mathcal{A}(f) \text {. }
$$

Proof. We infer from Remark 6.8 that

$$
\liminf _{i \rightarrow \infty} \mathcal{S}\left[\left(P_{R}^{k}: g_{i}\right), y\right] \geqq \mathcal{S}\left[\left(P_{R}^{k}: f\right), y\right] \quad \text { for } \quad R \in G_{n}, y \in E_{k},
$$

and apply Fatou's Theorem to the definition of $\mathcal{A}$.

6.12. THEOREM. If $X \subset E_{k}, f$ is a continuous function with domain $X$ to $E_{n}$, and if

then

$$
\limsup _{z \rightarrow x} \frac{|f(z)-f(x)|}{|z-x|}<\infty \quad \text { for } x \in \text { Interior } X \text {, }
$$

$$
\mathcal{A}(f)=\int_{E_{n}} N(f, \text { Interior } X, y) d \mathcal{L}_{n}^{k} y=\int_{\text {Interior } x} J f(x) d \mathcal{L}_{k}^{k} x .
$$

Proof. The theorem is a consequence of $6.9,4.5$, and the following proposition:

If $R \in G_{n}$, then

$$
\int_{E_{k}} S\left[\left(P_{R}^{k}: f\right), y\right] d \mathcal{L}_{k}^{k} y=\int_{E_{k}} N\left[\left(P_{R}^{k}: f\right), \text { Int } X, y\right] d \mathcal{L}_{k}^{k} y .
$$


To prove this statement, abbreviate $g=\left(P_{R^{k}}: f\right)$, and let $A$ be the set of all interior points of $X$ at which $g$ has a nonsingular differential.

First we note that

$$
S(g, y) \leqq N(g, \text { Int } X, y) \quad \text { for } y \in E_{k} \text {. }
$$

Next we infer from Theorem 6.4 that $g$ is stable at each point of $A$. As pointed out in 6.8 , this implies

$$
N(g, A, y) \leqq \delta(g, y)
$$

for $y \in E_{k}$.

We combine these inequalities with $[F 2,5.2]$, and the fact that $g$ is differentiable at $\mathcal{L}_{k}{ }^{k}$ almost all interior points of $X$ (see Saks, Theory of the integral, p. 310), to conclude

$$
\begin{aligned}
\int_{E_{k}} S(g, y) d \mathcal{L}_{k}^{k} y & \leqq \int_{E_{k}} N(g, \text { Int } X, y) d \mathcal{L}_{k}^{k} y \\
& =\int_{\operatorname{Int} x} J g(x) d \mathcal{L}_{k}^{k} x=\int_{A} J g(x) d \mathcal{L}_{k}^{k} x \\
& =\int_{E_{k}} N(g, A, y) d \mathcal{L}_{k}^{k} y \leqq \int_{E_{k}} S(g, y) d \mathcal{L}_{k}^{k} y .
\end{aligned}
$$

The proof is complete.

6.13. Remark. Fix $X$ and $F$ as in 6.8 . Then Theorem 6.11 is equivalent to the statement:

The function $A$ is lower semicontinuous on $F$.

Next let $D$ be the class of those functions $f$ which satisfy the hypotheses of Theorem 6.12, and let $I$ be the function on $D$ such that

$$
I(f)=\int_{\operatorname{Int} X} J f(x) d \mathcal{L}^{k} x \quad \text { for } \quad f \in D .
$$

The function $I$ is the "classical area integral." From 6.12 we know that

$$
I(f)=\mathcal{A}(f) \quad \text { for } f \in D .
$$

Accordingly, $I$ is lower semicontinuous on $D$, and $\mathcal{A}$ is a lower semicontinuous extension of $I$ over $F$.

Among all the lower semicontinuous extensions of $I$ over $F$ there is one, Lebesgue area, which has received particular attention in the literature. Lebesgue area is the function $L$ on $F$ such that

$$
L(f)=\liminf _{g \rightarrow, g \in D} I(g) \quad \text { for } f \in F .
$$

It follows immediately from this definition, and from the lower semicontinuity of $I$ on $D$, that Lebesgue area, $L$, is the numerically largest lower semicontinuous extension of $I$ over $F$. Hence, in particular, 


$$
\mathcal{A}(f) \leqq L(f)
$$

for $f \in F$.

Since $\mathcal{A}$ has an explicit topological meaning, and $L$ has proved useful in analysis because it permits approximation, in area, of continuous functions by differentiable functions, the problem of equality seems to be of interest. In 6.14 and 6.15 we shall give some incomplete results for the case of two-dimensional surfaces.

6.14. Remark. For nonparametric two-dimensional surfaces the problem of 6.13 is solved readily.

Let $X$ be an open rectangle, let $n=3$, define $F$ as in 6.8 , and let $f$ be a continuous numerically valued function on $E_{2}$. Recall the conventions of $[\mathrm{F} 1,6.1,5.2]$, and let

$$
g=(\bar{f} \mid X) .
$$

Then, we assert,

$$
A(g)=L(g)=H(f, X) .
$$

This follows from the first and last of the four statements below, whose proofs are only sketched.

Statement 1. $\mathcal{A}(g) \leqq L(g) \leqq H(f, X)$.

The first inequality was proved in 6.13 , whereas the second follows from the fact that $H(f, X)$ is the lower limit of the areas of nonparametric polyhedra approximating $g$ uniformly.

Statement 2. Recall [F1, 6.1]. Suppose $A$ is a bounded open convex subset of $E_{2}, i$ is a positive integer, and $R \in G_{3}$. Then

$$
\int_{E_{2}} N\left[\left(P_{R}^{2}: \bar{f}_{i}\right), A, y\right] d \mathcal{L}_{2}^{2} y \leqq i^{2} \pi^{-1} \int_{K_{i}} \int_{E_{2}} N\left[\left(P_{R}^{2}: \bar{f}\right), A_{z}, y\right] d \mathcal{L}_{2}^{2} y d \mathcal{L}_{2 z}^{2} .
$$

This can be proved by the methods of $[\mathrm{F} 1,6.2,6.3,6.5]$.

Statement 3. If $R \in G_{3}$, then

$$
N\left[\left(P_{R}^{2}: \bar{f}\right), X, y\right]=\mathcal{S}\left[\left(P_{R}^{2}: g\right), y\right] \text { for } \mathcal{L}_{2}^{2} \text { almost all } y \text { in } E_{2} .
$$

In view of the reduction procedure of $[\mathrm{F} 1,6.5]$, we assume $P_{R}{ }^{2}=P_{a}$ with $a \in E_{3},|a|=1, a_{1}=0, a_{2} \geqq 0, a_{3} \geqq 0$, and define, as in [F1, 6.2], the functions $h_{u}$ by the relation

$$
h_{u}(v)=a_{3} v-a_{2} g(u, v) \quad \text { for } \quad(u, v) \in E_{2} \text {. }
$$

Let $S$ be the set of all points $(u, v)$ such that $(u, v) \in X$ and $h_{u}$ has either a maximum or a minimum at $v$. Note that $S$ is of type $F_{\sigma}$, and that, for each number $u$, the set

$$
\underset{t}{E}\left[(u, t) \in\left(P_{a}: g\right)^{*}(S)\right]=h_{u}^{*}\{\underset{v}{E}[(u, v) \in S]\}
$$

is countable. Accordingly the set $\left(P_{a}: g\right)^{*}(S)$ is of type $F_{\varepsilon}$, with 


$$
\mathcal{L}_{2}^{2}\left\{\left[P_{a}: g\right]^{*}(S)\right\}=0,
$$

and the argument can be completed by showing that $\left(P_{a}: g\right)$ is stable at each point of $(X-S)$.

Pick $\left(u_{0}, v_{0}\right) \in(X-S)$ and $\delta>0$.

We can select numbers $u_{1}, u_{2}, v_{1}, v_{2}$ such that

$$
\begin{gathered}
u_{0}-\delta<u_{1}<u_{0}<u_{2}<u_{0}+\delta, \quad v_{0}-\delta<v_{1}<v_{0}<v_{2}<v_{0}+\delta, \\
Q=\underset{(u, v)}{E}\left[u_{1}<u<u_{2}, v_{1}<v<v_{2}\right] \subset X,
\end{gathered}
$$

and either

$$
\left[u_{1} \leqq u \leqq u_{2} \text { implies } h_{u}\left(v_{1}\right)<h_{u}\left(v_{0}\right)<h_{u}\left(v_{2}\right)\right]
$$

or else

$$
\left[u_{1} \leqq u \leqq u_{2} \text { implies } h_{u}\left(v_{1}\right)>h_{u}\left(v_{0}\right)>h_{u}\left(v_{2}\right)\right] \text {. }
$$

In each case the topological index of the point $\left[P_{a}: g\right]\left(u_{0}, v_{0}\right)$ with respect to the curve, which we get by confining the mapping $\left(P_{a}: g\right)$ to the boundary of the rectangle $Q$, is different from zero.

Following Radó (see [R1]), we infer that $\left(P_{a}: g\right)$ is stable at $\left(u_{0}, v_{0}\right)$.

Statement 4. $H(f, X) \leqq \mathcal{A}(g)$.

Choose an arbitrary rectangle $A$ whose closure is a subset of $X$. If $i$ is a positive integer, and so large that $A_{z} \subset X$ for $z \in K_{i}$, we infer from the Statements 2 and 3 that

$$
\begin{aligned}
H\left(f_{i}, A\right) & =\int_{A} J \bar{f}_{i}(x) d \mathcal{L}_{2}^{2} x \\
& =\int_{G_{3}} \int_{E_{2}} N\left[\left(P_{R}^{2}: \bar{f}_{i}\right), A, y\right] d \mathcal{L}_{2}^{2} y d \phi_{3} R \\
& \leqq \int_{G_{3}} i^{2} \pi^{-1} \int_{K_{i}} \int_{E_{2}} N\left[\left(P_{R}^{2}: \bar{f}\right), A_{2}, y\right] d \mathcal{L}_{2}^{2} y d \mathcal{L}_{2}^{2} z d \phi_{3} R \\
& \leqq \int_{G_{3}} \int_{E_{2}} N\left[\left(P_{R}^{2}: \bar{f}\right), X, y\right] d \mathcal{L}_{2}^{2} y d \phi_{3} R \\
& =\int_{G_{3}} \int_{E_{2}} S\left[\left(P_{R}^{2}: g\right), y\right] d \mathcal{L}_{2}^{2} y d \phi_{3} R=\mathcal{A}(g) .
\end{aligned}
$$

Consequently

$$
H(f, A)=\lim _{i \rightarrow \infty} H\left(f_{i}, A\right) \leqq \mathcal{A}(g)
$$

and Statement 4 follows from the arbitrary nature of $A$.

6.15. Remark. To discuss some aspects of the problem of 6.13 for twodimensional (parametric) surfaces, we specialize: 


$$
X=\underset{x}{E} \underset{E_{2}}{E}[|x| \leqq 1]
$$

The following result is due to Radó (see [R2]):

If $f \in F$ and $R \in G_{n}$, then $\left(P_{R}{ }^{2}: f\right)$ is stable at $\mathcal{L}_{2}{ }^{2}$ almost all those points $x$ in $X$ at which $f$ has finite partial derivatives and $J\left(P_{R^{2}}: f\right)(x)>0$.

By an argument similar to the proof of Theorem 6.12, in which the preceding statement replaces Theorem 6.4 , and $[F 2,6.1]$ replaces $[F 2,5.2]$, we arrive at the following proposition:

If $f \in F$ and $Q$ is the set of those points of $X$ at which has finite partial derivatives, then

$$
A(f) \geqq \int_{Q} J f(x) d \mathcal{L}_{2}^{2} x .
$$

[If (Interior $X) \subset Q$, then equality holds.]

Since $L$ and $\mathcal{A}$ are invariant under Fréchet equivalence (see [R1], [Y]) we conclude:

If $f \in F$, and there exists such $a g \in F$ that $f$ and $g$ are Fréchet equivalent, and $\dot{g}$ has finite partial derivatives $\mathcal{L}_{2}{ }^{2}$ almost everywhere in $X$ with

$$
L(g)=\int_{X} J g(x) d \mathcal{L}_{2}^{2} x
$$

then

$$
\mathcal{A}(f)=L(f) .
$$

A great deal of research has been devoted to conditions under which the hypothesis of the last proposition holds (see [M], [McS], [R]). There is a close relation between the theories of stable values, and of essential multiplicity of Radó and Reichelderfer. Using their notation we have, in fact, the equality

$$
S(T, y)=\kappa(y, T, X) \quad \text { for } y \in E_{2} \text {, }
$$

whenever $T$ is a continuous function $\left[\operatorname{such}\right.$ as $\left.\left(P_{R^{2}}: f\right)\right]$ on the $\operatorname{disc} X$ to the plane $E_{2}$ (see $[R R, 1.11,1.17]$ ).

\section{BIBLIOGRAPHY}

A. S. Besicovitch

[BE] On the fundamental geometrical properties of linearly measurable plane sets of points (III), Math. Ann. vol. 116 (1939).

W. BLASCHKE

[B] Vorlesungen ïber Integralgeometrie. Hamburger Mathematisch Einzelschriften vol. 20 (1936) and vol. 22 (1937).

H. FEDERER

[F1] Surface area. I. Trans. Amer. Math. Soc. vol. 55 (1944).

[F2] Surface area. II. Trans. Amer. Math. Soc. vol. 55 (1944).

[F3] The Gauss-Green Theorem. Trans. Amer. Math. Soc. vol. 58 (1945). 
W. HuREWicz and H. WALlman

[HW] Dimension theory, Princeton Mathematical Series, vol. 4, 1941.

E. J. McShane

[McS] Integrals over surfaces in parametric form, Ann. of Math. vol. 34 (1933).

C. B. MORREY, JR.

[M1] A class of representations of manifolds, Amer. J. Math. vol. 55 (1933) and vol.56 (1934)

[M2] An analytic characterization of surfaces of finile Lebesgue area, Amer. J. Math. vol. 57 (1935) and vol. 58 (1936).

A. P. Morse and H. Federer

[MF] Some properties of measurable functions, Bull. Amer. Math. Soc. vol. 49 (1943).

A. P. MoRse AND J. F. RANDOLPH

[MR] The $\phi$ rectifiable subsets of the plane, Trans. Amer. Math. Soc. vol. 55 (1944).

H. PoIncare

[P] Calcul des probabilites, Paris, Gautiers-Villars, 1912.

T. RADó

[R1] Über das Flächenmass rektifizierbarer Flächen, Math. Ann. vol. 100 (1928).

[R2] On the derivative of the Lebesgue area of continuous surfaces, Fund. Math. vol. 30 (1938).

[R3] On the semicontinuity of double integrals in parametric form, Trans. Amer. Math. Soc. vol. 51 (1942).

T. RAD6 AND P. REICHelderfer

[RR] $A$ theory of absolutely continuous transformations in the plane, Trans. Amer. Math. Soc. vol. 49 (1941).

L. A. Santal 6

[S] Integralgeometrie 5. Über das kinematische Mass im Raum, Actualités scientifiques et industrielles, 1935.

S. Sherman

[SH] A comparison of linear measures in the plane, Duke Math. J. vol. 9 (1942).

A. WEIL

[W] L'intégration dans les groupes topologigues et ses applications, Actualités scientifiques et industrielles, 1938.

J. W. T. Youngs

[Y] The topological theory of Fréchet surfaces, Ann. of Math. vol. 45 (1944).

Ballistic REsearch Laboratory, Aberdeen Proving Ground, Md. 